\title{
Correction to: Surface-enhanced infrared detection of benzene in air using a porous metal-organic-frameworks film
}

\author{
Raekyung Kim ${ }^{*, \ddagger}$, Seohyeon Jee ${ }^{*, \ddagger}$, Unjin Ryu* ${ }^{*}$ Hyeon Shin Lee*, Se Yun Kim ${ }^{* *, \dagger}$, and Kyung Min Choi ${ }^{*, \dagger}$ \\ *Department of Chemical and Biological Engineering, Sookmyung Women's University, \\ 100 Cheongpa-ro 47 gil, Yongsan-gu, Seoul 04310, Korea \\ **Department of Materials Science \& Engineering, KAIST, Daejeon 34141, Korea \\ (Received 31 October 2018 - accepted 17 January 2019)
}

The article Surface-enhanced infrared detection of benzene in air using a porous metal-organic-frameworks film, written by Raekyung $\mathrm{Kim}^{* * *}$, Seohyeon Jee**, Unjin Ryu*, Hyeon Shin Lee*, Se Yun $\mathrm{Kim}^{* *, \dagger}$, and Kyung Min Choi ${ }^{*, \dagger}$, was originally published on the publisher's internet portal (currently SPringerLink) on 18 Febru- ary 2019 with misprinted DOI number, 10.1007/s11814-018-02310 , due to the technical error from converting manuscript file from Microsoft Word to PDF. The correct DOI number for the article is 10.1007/s11814-019-0315-X.

${ }^{\dagger}$ To whom correspondence should be addressed.

E-mail: ksyvip@kaist.ac.kr, kmchoi@sookmyung.ac.kr

${ }^{*}$ These authors contributed equally.

Copyright by The Korean Institute of Chemical Engineers. 